\title{
Applicability of a Schmidt law to star formation in ring galaxies
}

\author{
E. I. Vorobyov ${ }^{\star}$ \\ Institute of Physics, Stachki 194, Rostov-on-Don, Russia, and Isaac Newton Institute of Chile, Rostov-on-Don Branch, Russia \\ Received 26 March 2003 / Accepted 19 June 2003

\begin{abstract}
The gas hydrodynamics modeling of the Cartwheel ring galaxy is performed with the purpose to reproduce the measured intensity and radial distribution of $\mathrm{H} \alpha$ emission in the Cartwheel's disk. The star formation efficiency of $\epsilon=0.09-0.18$ is derived for an assumed Schmidt law with power index $N=1.5$ and a full thickness of the Cartwheel's gas disk of $z_{0}=250-1000 \mathrm{pc}$, respectively. Simulations with a Schmidt law of star formation yield a higher $\mathrm{H} \alpha$ surface brightness $\left(\Sigma_{\mathrm{H} \alpha}\right)$ in the Cartwheel's inner ring as compared to the observed values. The Toomre criterion for star formation fails to account for the observed sharp drop of massive star formation (MSF) in the inner ring. Numerical simulations indicate that a large shear near/at the position of the inner ring can raise the gas threshold for star formation and suppress MSF in the Cartwheel's inner regions. Hence, the Schmidt law has to be supplemented with a shear criterion for star formation in order to reproduce the observed radial distribution of $\Sigma_{\mathrm{H} \alpha}$ in the Cartwheel's inner regions.
\end{abstract}

Key words. galaxies: individual: the Cartwheel - galaxies: starburst

\section{Introduction}

Collisional ring galaxies provide the means of studying star formation processes on a kiloparsec scale. The gravitational perturbation of a companion galaxy drives a ring density wave through the disk of the target galaxy. The expanding-ring density wave is expected to trigger high rates of massive star formation (MSF) along its perimeter. On the other hand, strong suppression of star formation is expected behind the wave where the surface density of the gas drops substantially below the initial unperturbed values. $\mathrm{H} \alpha$ imaging of a sample of ring galaxies by Marston \& Appleton (1995) and Higdon (1995) has demonstrated that in most ring galaxies star formation is indeed localized in the rings.

Observations of both normal and starburst disk galaxies suggest that on scales of a few kiloparsecs star formation may be well represented by a Schmidt law with index $N=$ $1.4 \pm 0.15$ (Kennicutt 1998). Can star formation in ring galaxies, particularly in the Cartwheel ring galaxy, be described by a Schmidt law as well? A general correlation between the azimuthally averaged radial profiles of the $\mathrm{H}$ I surface density (hereafter, $\Sigma_{\mathrm{HI}}$ ) and the $\mathrm{H} \alpha$ surface brightness (hereafter, $\Sigma_{\mathrm{H} \alpha}$ ) in the Cartwheel's disk seems to favor a global Schmidt law. However, a pronounced anticorrelation between the azimuthal distributions of $\Sigma_{\mathrm{HI}}$ and $\Sigma_{\mathrm{H} \alpha}$ found by Higdon (1996) in the Cartwheel's outer ring implies that the Schmidt law is not locally observed. Further, emission-line maps of the Cartwheel (Amram et al. 1998; Vorobyov \& Bizyaev 2003, hereafter VB) suggest that the inner ring is at best much weaker than the outer ring in terms of MSF. This is unexpected, since numerical

* e-mail: eduard_vorobev@mail.ru simulations of the Cartwheel predict gas surface densities in the inner ring exceeding those of the outer ring (Struck-Marcell \& Higdon 1993; VB). Low-level star formation activity in the regions of high gas surface density implies that there may exist a mechanism or mechanisms suppressing the MSF in the Cartwheel's inner ring.

\section{Star formation in the Cartwheel}

Figure 1 shows the $\mathrm{H} \alpha$ image of the Cartwheel galaxy obtained from the CFHT archive (VB). Since this image was intended to show the relative input of different parts of the Cartwheel to the total $\mathrm{H} \alpha$ emission, the flux calibration was not applied. The contrast in the $\mathrm{H} \alpha$ image is enhanced to show faint lineemitting regions in the inner ring. The $\mathrm{H} \alpha$ emission is mainly restricted to the outer ring. The inner ring shows much fainter $\mathrm{H} \alpha$ emission. The $\mathrm{H} \alpha$ luminosity of the Cartwheel was found to be $1.7 \times 10^{42} \mathrm{ergs} \mathrm{s}^{-1}$ (Higdon 1995). At the distance of $140 \mathrm{Mpc}\left(H=65 \mathrm{~km} \mathrm{~s}^{-1} \mathrm{Mpc}^{-1}\right)$ assumed in this paper, it becomes $4 \times 10^{42} \mathrm{ergs} \mathrm{s}^{-1}$.

The $\mathrm{H} \alpha$ emission line provides a measure of the star formation rate (SFR) over the past 6-7 Myr. Calibrations have been published by several authors, including Gallagher et al. (1984), Kennicutt et al. (1994), and Leitherer \& Heckman (1995). There is a $30 \%$ variation among published calibrations, with most of the dispersion reflecting differences in stellar evolution and atmospheric models. In this paper the calibration of Kennicutt et al. (1994) derived for solar metallicity and the Salpeter IMF $\left(0.1-100 M_{\odot}\right)$ is used:

$\operatorname{SFR}\left(M_{\odot} \mathrm{yr}^{-1}\right)=7.9 \times 10^{-42} L_{\mathrm{H} \alpha}\left(\operatorname{ergs~s}^{-1}\right)$. 


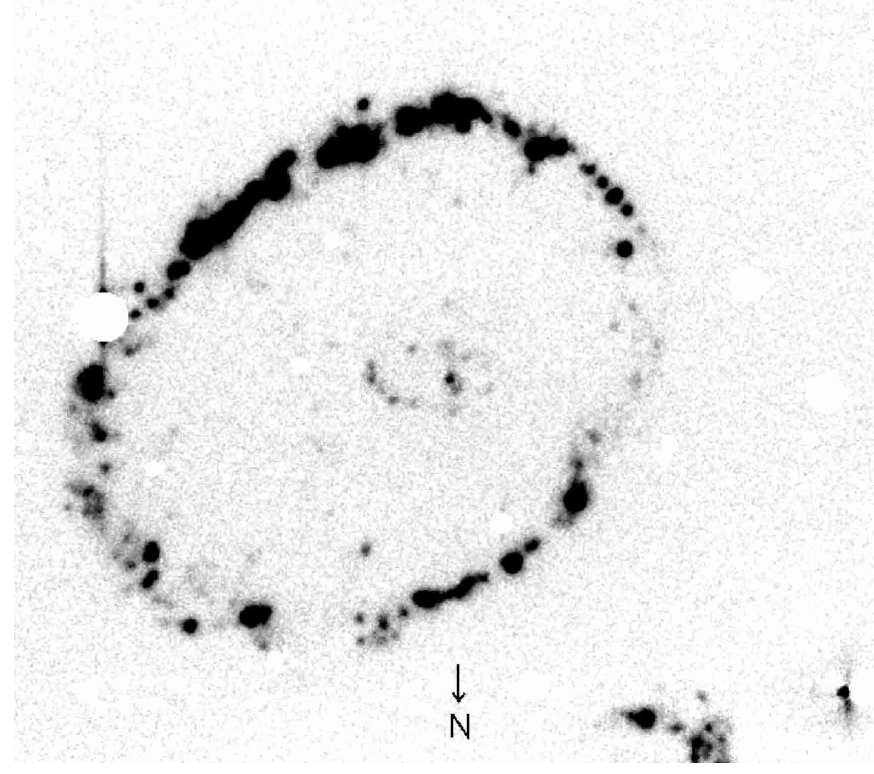

Fig. 1. The Cartwheel galaxy in $\mathrm{H} \alpha$. The north is shown by the arrow.

Metal-poor stars produce more ionizing photons than metalrich stars. A population synthesis exercise with the Starburst99 code (Leitherer et al. 1999) indicates that the conversion factor in Eq. (1) is $20 \%$ lower for the sub-solar metallicity of $z_{\odot} / 5$.

Actually, the largest source of uncertainty in Eq. (1) is the amount of internal extinction at $\mathrm{H} \alpha, A_{\mathrm{H} \alpha}=0.75 A_{\mathrm{v}}$ (Cardelli et al. 1989). In the present simulations, a possible differential extinction in the Cartwheel's disk is taken into account by estimating $A_{\mathrm{v}}$ from the model's known gas surface density, $\Sigma_{\text {gas }}$, via the standard gas-to-dust ratio (Bohlin et al. 1978) and $R_{\mathrm{v}}=3.1$ (see Vorobyov \& Bizyaev 2001 for details).

Observations of both normal and starburst disk galaxies suggest that on the scales of a few kiloparsecs star formation may be represented by a Schmidt law (Kennicutt 1998)

$\Sigma_{\mathrm{SFR}}=(2.5 \pm 0.7) \times 10^{-4} \Sigma_{\mathrm{gas}}^{1.4 \pm 0.15}$,

where $\Sigma_{\mathrm{SFR}}\left(M_{\odot} \mathrm{yr}^{-1} \mathrm{kpc}^{-2}\right)$ is the star formation rate per unit area and $\Sigma_{\text {gas }}\left(M_{\odot} \mathrm{pc}^{-2}\right)$ is the gas surface density. As discussed by Elmegreen (1997), a Schmidt law with index $N \sim 1.5$ would be expected for self-graviting disks, if the SFR is equal to the ratio of the local gas volume density $(\rho)$ to the free-fall time $\left(\propto \rho^{-0.5}\right)$, all multiplied by some efficiency $\epsilon$. This results in

$\Sigma_{\mathrm{SFR}}=0.12 \epsilon_{z 0} \Sigma_{\text {gas }}^{1.5}$,

where $\epsilon_{z 0} \equiv \epsilon z_{0}^{-0.5}$ and $z_{0}(\mathrm{pc})$ is the full width of the gas disk. The efficiency $\epsilon$ is a measure of the fraction of the gas mass converted into stars before the clouds are disrupted. Neglecting a small difference in the exponent, the efficiency of star formation in Kennicutt's sample of galaxies becomes $\epsilon=0.02-0.08$ for the adopted $z_{0}=250-1000 \mathrm{pc}$, respectively. In the following sections, the Cartwheel's $\mathrm{H} \alpha$ intensity and radial distribution are modeled and the resulting efficiency of star formation is determined.

\section{The numerical model}

The numerical hydrodynamics model used to simulate the gas dynamics in the Cartwheel is discussed in detail in VB. Two modifications are made as compared to the code described in VB. First, a gravitational potential of the pre-collision rigid stellar disk is added. Second, $\Sigma \mathrm{H} \alpha$ distribution is constructed using Eq. (1). To compute the SFR, stellar particles are formed according to the Schmidt law (Eq. 3) each $0.5 \mathrm{Myr}$ in each computational cell. The average mass of a stellar particle is $10^{3} M_{\odot}$, with the maximum mass amounting to $0.5 \times 10^{5} M_{\odot}$ and the minimum mass as small as the mass of a single massive star. Further, stellar particles are assigned positions and velocities drawn from the parent gas and are evolved as collisionless particles in the combined gravitational potential of the halo, stellar disk, and gas disk. The local self-gravity among stellar particles is not computed. Since each stellar particle carries information on the SFR at the time of its formation, the SFR averaged over the past $7 \mathrm{Myr}$ in each cell is easily constructed, and then substituted in Eq. (1) to obtain $\Sigma_{\mathrm{H} \alpha}$. The conversion factor in Eq. (1) decreases linearly from $7.9 \times 10^{-42}$ in the nucleus to $6.3 \times 10^{-42}$ in the outer ring to account for the radial metallicity gradient ranging from $z_{\odot}$ in the inner regions $(R<6 \mathrm{kpc})$ to $z_{\odot} / 5$ in the outer regions (Vorobyov \& Bizyaev 2001; Fosbury $\&$ Hawarden 1977). The gas consumption due to star formation is taken into account. Possible feedback effects of star formation on the system are neglected in the present simulations.

The parameters of the numerical simulations are mostly identical to those in VB. A few modifications are made, namely the halo mass is reduced to $4 \times 10^{11} M_{\odot}$ because of the inclusion of the stellar disk of $1.0 \times 10^{11}$, so that the total halo+stellar mass is comparable to the indicative mass of the Cartwheel, $5.3 \times 10^{11} M_{\odot}$ (Higdon 1996). The total gas mass is increased from $3.0 \times 10^{10} M_{\odot}(\mathrm{VB})$ to $3.5 \times 10^{10} M_{\odot}$ to take account of $M_{\mathrm{H}_{2}}=2 \times 10^{9} M_{\odot}$ detected by Horellou et al. (1998) in the Cartwheel's inner regions and a possible contribution of molecular hydrogen in the star-forming outer ring. In order to make unambiguous conclusions about the applicability of a Schmidt law for describing star formation in ring galaxies, the gas distribution in the Cartwheel's disk has to be modeled as accurately as possible. Several test runs have shown that the Cartwheel's H I kinematics and morphology are best reproduced for an off-center collision with $R_{\text {impact }}=4 \mathrm{kpc}$, in contrast to $R_{\text {impact }}=3 \mathrm{kpc}$ in VB. The companion's mass is chosen to be $2.0 \times 10^{11} M_{\odot}$ in the present simulations. This mass is about 5 times larger than what is actually measured by Davies \& Morton (1982) and Higdon (1996) for a companion G3, the most massive in the Cartwheel group. Most studies indicate that the well-defined rings are formed only for companions with masses no less than $20 \%$ of the primary galaxy's mass (see e.g. Hernquist \& Wail 1993). However, there is good reason to believe that the "missing" mass problem is a consequence of the ring galaxy formation (see discussion in VB).

\section{The model $\mathrm{H} \alpha$ intensity and radial distribution}

The present study addresses two main questions:

1) What is the star formation efficiency $(\epsilon)$ in the Cartwheel? 
2) Which modifications to the Schmidt law are required to reproduce the observed azimuthally averaged $\Sigma_{\mathrm{H} \alpha}$ distribution in the Cartwheel?

This approach is different from that of Mihos \& Hernquist (1994), who fixed $\epsilon$ so that their model galaxy formed stars at roughly a rate of $1 M_{\odot} \mathrm{yr}^{-1}$.

\subsection{An isothermal equation of state}

Firstly, an isothermal equation of state is considered with the gas temperature $10^{4} \mathrm{~K}$. The coefficient $\epsilon_{z 0}$ in Eq. (3) is varied to obtain the observed value for the integrated $\mathrm{H} \alpha$ luminosity, $4.0 \times 10^{42} \mathrm{ergs} \mathrm{s}^{-1}$. This is achieved for $\epsilon_{z 0} \equiv \epsilon z_{0}^{-0.5}=$ $5.7 \times 10^{-3}$. Assuming that the full thickness of the Cartwheel's gas disk, $z_{0}$, is in the range of $250-1000 \mathrm{pc}$, the efficiency of star formation becomes $\epsilon=0.09-0.18$, respectively. Note that numerical simulations (Struck 1997), as well as observations (VB), indicate that the Cartwheel's disk is rather thick. Either star formation in the Cartwheel is very efficient or the $\mathrm{H} \alpha$ luminosity reported by Higdon (1995) is overestimated.

In the following simulations, $z_{0}=500 \mathrm{pc}$ or $\epsilon=0.13$ is assumed. The solid line in Fig. 2 shows the radial $\Sigma_{\mathrm{H} \alpha}$ profile obtained by azimuthally averaging the modeled $\Sigma_{\mathrm{H} \alpha}$ around the dynamical center at $r=0 \mathrm{kpc}$. The dotted line gives the observed $\mathrm{H} \alpha$ radial profile of the Cartwheel derived from Fig. 1 (see VB for details). Since the flux calibration was not originally applied to Fig. 1, the Cartwheel's $\Sigma_{\mathrm{H} \alpha}$ radial profile in Fig. 2 was calibrated to the integrated $\mathrm{H} \alpha$ luminosity of the Cartwheel, $L_{\mathrm{H} \alpha}=4.0 \times 10^{42} \mathrm{ergs} \mathrm{s}^{-1}$. Simulations with a Schmidt law of star formation predict a higher or comparable $\Sigma_{\mathrm{H} \alpha}$ in the inner ring $(r<6 \mathrm{kpc})$ as compared to that of the outer ring, while the Cartwheel's inner ring is an order of magnitude weaker in terms of $\Sigma_{\mathrm{H} \alpha}$. This discrepancy is not due to gas-density-dependent extinction in the Cartwheel's disk, this effect is accurately taken into account as described in Sect. 2. There should exist a mechanism or mechanisms that suppress star formation in the Cartwheel's inner regions.

Higdon (1996) has suggested that a gas threshold effect described by the Toomre criterion is responsible for the lack of MSF interior to the Cartwheel's outer ring. As found by Kennicutt (1989) and confirmed by Martin \& Kennicutt (2001), MSF occurs only if $\Sigma_{\text {gas }}>\Sigma_{\text {crit }}=0.7 v_{\mathrm{s}} k / G \pi$, where $v_{\mathrm{s}}$ is the speed of sound and $k$ is the epicyclic frequency. The simulations are repeated with a Toomre-modified Schmidt law, i.e. star formation takes place only if the local $\Sigma_{\text {gas }}>\Sigma_{\text {crit }}$, with $v_{\mathrm{s}}=8 \mathrm{~km} \mathrm{~s}^{-1}$ and $k$ computed from the model's known velocity field. The results are shown in Fig. 2 by the dashed line. The Toomre criterion affects $\Sigma_{\mathrm{H} \alpha}$ only in the inter-ring region $(6<r<20 \mathrm{kpc})$ where $\Sigma_{\text {gas }}$ drops below critical values and has almost no influence on $\Sigma_{\mathrm{H} \alpha}$ in the inner and outer rings.

Another mechanism that might suppress star formation is a large shear. The effects of shear can be parameterized in terms of the free-fall time defined as $t_{\mathrm{ff}}=\left(3 \pi z_{0} / 32 G \Sigma_{\text {gas }}\right)^{0.5}$, and the local shear rate defined as $0.5 r \mathrm{~d} \Omega / \mathrm{d} r$. Star formation is supposed to occur only if the following condition is met $0.5 r \mathrm{~d} \Omega / \mathrm{d} r<1 / t_{\mathrm{ff}}$. This inequality simply states that a gas cloud is stable against the shear if the time it takes for the

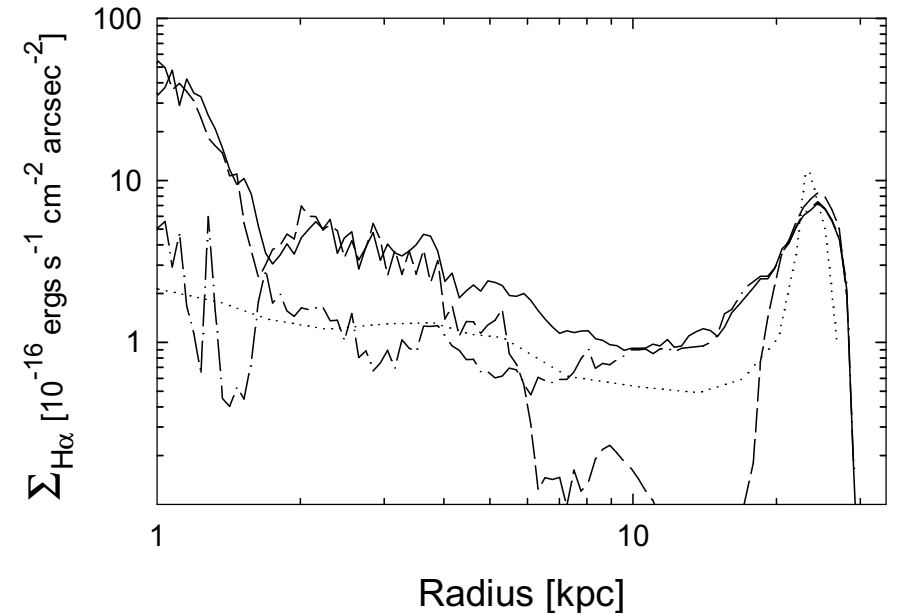

Fig. 2. The azimuthally averaged $\Sigma_{\mathrm{H} \alpha}$ distributions obtained for a pure Schmidt law (the solid line), the Schmidt law supplemented with the Toomre criterion for star formation (the dashed line), and the Schmidt law supplemented with the shear criterion (the dashed-dotted line). The dotted line represents the observed $\Sigma_{\mathrm{H} \alpha}$ radial profile.

shear to tear apart a cloud is bigger than the free-fall time. Solving this inequality for $\Sigma_{\text {gas }}$ and noticing that the expression $-0.5 r \mathrm{~d} \Omega / \mathrm{d} r$ is in fact the Oort constant $A$ results in the following shear criterion for star formation

$\Sigma_{\text {gas }}>\Sigma_{\text {crit }}^{\text {shear }}=\frac{\mu A^{2} z_{0}}{\pi G}$,

where $\mu$ is a constant of order unity.

The shear criterion (4) is taken into account when deriving the azimuthally averaged $\Sigma_{\mathrm{H} \alpha}$ shown in Fig. 2 by the dotteddashed line. The Oort constant $A$ is computed from the model's known velocity field. A Schmidt law supplemented with the shear criterion (4) yields an azimuthally averaged $\Sigma_{\mathrm{H} \alpha}$ that agrees better with the observed profile of the inner ring, which proves that the shear could be effective in suppressing MSF in the Cartwheel's inner region. On the other hand, the shear appears to have little effect on star formation in the Cartwheel's outer regions.

Substituting $z_{0}=\sigma^{2} / \pi G \Sigma_{\text {gas }}$ in Eq. (4) results in another definition of the critical density $\Sigma_{\text {crit }}^{\text {shear }}=\alpha A \sigma / \pi G$, where $\sigma$ is a one-dimensional velocity dispersion and $\alpha \approx 1$. This definition of $\Sigma_{\text {crit }}^{\text {shear }}$ is outlined in Martin \& Kennicutt (2001) who refer to Elmegreen (1993) and Hunter et al. (1998), the only difference is in the value of $\alpha=2.5$. As discussed in Hunter et al. (1998), the coefficient $\alpha=2.5$ is derived from the assumption that the amplitude of shearing perturbations must grow by a sufficiently large factor, $\sim 100$, during the characteristic time of $2 / A$ for the gravitational instability to be significant. If the gas disk of the Cartwheel was marginally stable before the collision (the high star formation efficiency favors this assumption), then a smaller increase in $\Sigma_{\text {gas }}$, say $\sim 10$, might be enough for the instability to grow, which would result in $\alpha=1$. Simulations with different values of $\alpha$ indicate that $\alpha=1$ reproduces best the observed azimuthally averaged $\Sigma_{\mathrm{H} \alpha}$ profile of the Cartwheel's inner regions.

Recently, Martin \& Kennicutt (2001) have demonstrated that the shear may play an important role in determining a gas 
threshold for star formation in the inner parts of NGC 2403 and M 33, whose gas surface densities are well below $\Sigma_{\text {crit }}$, yet they have numerous H II regions. Indeed, if the local shear rate, rather than the Coriolis force (epicyclic frequency $k$ ), best describes the destruction rate of gas clouds, then the inner parts of galaxies with slowly rising rotation curves should have a lower gas threshold than the outer parts with flat rotation curves. It appears that the shear in the strongly perturbed inner parts of ring galaxies has the opposite effect on a gas threshold for star formation. Simulations show that the velocity field at/near the inner ring is strongly perturbed as a result of radial cross-motions of gas, which may effectively raise the gas threshold and suppress star formation there.

\subsection{An adiabatic equation of state with cooling and heating}

Simulations of Sect. 4.1 were repeated for an adiabatic equation of state including the effect of cooling and heating. The gas disk was initially set at $T=10^{4} \mathrm{~K}$. The equilibrium cooling curves of Wada \& Norman (2001) (their Fig. 1) were adopted. The cooling function is parameterized in terms of metallicity, i.e. it linearly decreases from solar metallicity in the nucleus to one-fifth of solar in the outer ring. An empirical heating function that initially balances the cooling is applied. This approach may be thought of as a crude model for stellar energy input and can imitate a constant heating of $\sim 10^{-24} \mathrm{ergs} \mathrm{s}^{-1}$ by the background UV radiation field. Cooling and heating were treated numerically using Newton-Raphson iterations.

The star formation efficiency in the non-isothermal gas depends on the gas temperature. The relevant physics is poorly understood and different modifications based on empirical relations have been proposed to account for the gas temperature dependence of a Schmidt law (e.g. Samland et al. 1997). To be consistent with the isothermal simulations in Sect. 4.1, the Schmidt law is modified so that the SFR is equal to that for the isothermal gas at $T<10^{4} \mathrm{~K}$ and rapidly declines at $T>10^{4} \mathrm{~K}$. The modified Schmidt law can now be written as $\Sigma_{\mathrm{SFR}}=0.12 \mu \epsilon_{\mathrm{z} 0} \Sigma_{\text {gas }}^{1.5}$, where

$\mu=\left\{\begin{array}{cc}{\left[\frac{2 \times 10^{4}(\mathrm{~K})}{10^{4}(\mathrm{~K})+T_{\text {gas }}}\right]^{2}} & \text { for } T_{\text {gas }}>10^{4} \mathrm{~K} \\ 1.0 & \text { for } T_{\text {gas }}<10^{4} \mathrm{~K} .\end{array}\right.$

Simulations indicate that the gas dynamics and morphology are similar on large scales in both isothermal and non-isothermal cases; the result is concurrent with that of Struck (1997). The gas in the outer ring is mostly found at $T=0.3-1.0 \times 10^{4} \mathrm{~K}$. It never cools below $0.2 \times 10^{4} \mathrm{~K}$, which partly explains why the ISM in the Cartwheel's outer ring is mainly found in the atomic phase. Contrary to the intuitive expectations, the gas in the strong inner ring is not shock-heated above $1.0 \times 10^{4} \mathrm{~K}$. Instead, high-density clumps $\left(50<\Sigma_{\text {gas }}<100 M_{\odot}\right)$ are found in the inner ring with temperatures as low as $150 \mathrm{~K}$. Development of such low-temperature, high-density clumps is interesting, since high densities and low temperatures are thought to favor $\mathrm{H} \mathrm{I} \rightarrow \mathrm{H}_{2}$ transitions in giant gas clouds. The inter-ring region is filled with a warm $T=1.0-2 \times 10^{4} \mathrm{~K}$, rarefied gas, hardly capable of forming stars.

The model $\mathrm{H} \alpha$ distribution is found to be similar to that of the isothermal case if an unmodified Schmidt law is employed, the azimuthally averaged $\Sigma_{\mathrm{H} \alpha}$ of the inner regions is higher or comparable to that of the outer ring. However, the measured azimuthally averaged $\Sigma_{\mathrm{H} \alpha}$ profile of the Cartwheel's inter-ring region $(10<r<20 \mathrm{kpc})$ is better reproduced in non-isothermal simulations, indicating that the temperature dependence of a Schmidt law rather than the Toomre criterion is responsible for the observed low-level star formation activity in the inter-ring region.

Thus, simulations with the effects of cooling and heating show that shocks and compressional heating are not effective in preventing MSF in the inner ring. The characteristic cooling times in the inner ring are still shorter than the gas dynamical times. A better correspondence between the modeled and observed azimuthally averaged $\Sigma_{\mathrm{H} \alpha}$ in the inner regions $(r \leq 6 \mathrm{kpc})$ can only be achieved for a Schmidt law supplemented by the shear criterion for star formation defined in Eq. (4). Hence, both isothermal and non-isothermal simulations indicate that the large shear may prevent MSF in the Cartwheel's inner ring.

\section{Conclusions}

Gas hydrodynamics modeling and a Schmidt law of star formation are used to simulate the total $\mathrm{H} \alpha$ luminosity from the Cartwheel ring galaxy, $L_{\mathrm{H} \alpha}=4.0 \times 10^{42} \mathrm{ergs} \mathrm{s}^{-1}$. The efficiency of star formation $\epsilon=0.09-0.18$ is derived for the assumed full thickness of the Cartwheel's gas disk of $z_{0}=250-1000 \mathrm{pc}$, respectively. The corresponding efficiency in Kennicutt's sample of isolated and interacting galaxies (Kennicutt 1998) is $\epsilon=0.02-0.08$, which implies that star formation in the Cartwheel is very efficient. Indeed, according to Marston \& Appleton (1995) the Cartwheel galaxy has an exceptional $L_{\mathrm{H} \alpha}$, at least 6 times higher than $L_{\mathrm{H} \alpha}$ of the other 11 ring galaxies in their sample. The reasons for such a star formation activity are uncertain. A more accurate determination of the Cartwheel's total $\mathrm{H} \alpha$ luminosity is highly desired to prove the exceptional status of this galaxy.

Simulations with a Schmidt law of star formation predict higher or comparable azimuthally averaged $\Sigma_{\mathrm{H} \alpha}$ in the inner ring than in the outer ring. Observations indicate the opposite, the Cartwheel's inner ring is much weaker in terms of $\Sigma_{\mathrm{H} \alpha}$. Simulations with the effects of cooling and heating show that the shocks and compressional heating are not effective in preventing MSF in the inner ring. Instead, high-density $50<\Sigma_{\text {gas }}<100 M_{\odot}$ and low-temperature $150<T<400 \mathrm{~K}$ clumps are formed in the inner ring, which may favor $\mathrm{H} \mathrm{I} \rightarrow \mathrm{H}_{2}$ transitions and account for the large amount of molecular hydrogen detected by Horellou et al. (1998) in the Cartwheel's inner regions. A Schmidt law supplemented with the Toomre criterion for star formation cannot explain a sharp drop of star formation in the inner ring. Failure of the Toomre criterion is also reported by Martin \& Kennicutt (2001) for NGC 2403 and M 33 where there is evidence for strong radial flows of gas. With radial gas velocities between $60 \mathrm{~km} \mathrm{~s}^{-1}$ (Higdon 1996) 
and $100 \mathrm{~km} \mathrm{~s}^{-1}(\mathrm{VB})$, the Cartwheel appears a classic case of failure of the Toomre criterion.

Numerical simulations indicate that the large shear near/at the position of the inner ring may raise the gas threshold for star formation and effectively suppress MSF in the inner ring. Thus, the Schmidt law has to be supplemented with the shear criterion for star formation in order to reproduce the measured azimuthally averaged $\Sigma_{\mathrm{H} \alpha}$ profile in the Cartwheel's inner regions $(r<6 \mathrm{kpc})$. The existence of large shear in the Cartwheel's inner regions is observationally supported by the detection of strong perturbations in the $\mathrm{H} \alpha$ rotation curve near/at the position of the inner ring (Amram et al. 1998). The measured azimuthally averaged $\Sigma_{\mathrm{H} \alpha}$ profile of the Cartwheel's inner regions is best reproduced for a 2.5 times lower $\Sigma_{\text {crit }}^{\text {shear }}$ than that outlined in Martin \& Kennicutt (2001) (refering to Elmegreen 1993 and Hunter et al. 1998). The difference implies that the gas disk of the Cartwheel was marginally stable before the collision.

Acknowledgements. The author is thankful to the anonymous referee for suggestions that helped to improve the paper. The author is grateful to D. Bizyaev for providing the $\mathrm{H} \alpha$ image of the Cartwheel galaxy.

\section{References}

Amram, O., Mendes de Oliveira, C., Boulesteix, J., \& Balkowaki, C. 1998, A\&A, 330, 881

Bohlin, R. C., Savage, B. D., \& Drake, J. F. 1978, ApJ, 224, 225

Cardelli, J. A., Geoffrey, C. C., \& Mathis, J. S. 1989, ApJ, 345, 245
Davies, R. L., \& Morton, D. C. 1982, MNRAS, 201, 69P

Elmegreen, B. 1993, in Star Formation, Galaxies and the Interstellar Medium, ed. J. Franco, F. Ferrini, \& G. Tenorio-Tagle (Cambridge, Cambrigde Univ. Press), 337

Elmegreen, B. 1997, in Starburst Activity in Galaxies, ed. J. Franco, R. Terlevich, \& A. Serrano, Rev. Mex. Astron. Astrophys. Conf. Ser., 6,165

Fosbury, R. A. E., \& Hawarden, T. G. 1977, MNRAS, 178, 473

Gallagher, J. S., Hunter, D. A., \& Tutukov, A. V. 1984, ApJ, 284, 544

Hernquist, L., \& Wail, M. L. 1993, MNRAS, 261, 804

Higdon, J. L. 1995, ApJ, 455, 524

Higdon, J. L. 1996, ApJ, 467, 241

Horellou, G., Charmandaris, V., Combes, F., et al. 1998, A\&A, 340, L51

Hunter, D., Elmegreen, B. G., \& Baker A. L. 1998, ApJ, 493, 595

Kennicutt, R. C. 1989, ApJ, 344, 658

Kennicutt, R. C., Tamblyn, P., \& Congdon, C. W. 1994, ApJ, 435, 22

Kennicutt, R. C. 1998, ApJ, 498, 541

Leitherer, C., \& Heckman, T. M. 1995, ApJS, 96, 9

Leitherer, C., Schaerer, D., Goldader, J. D., et al. 1999, ApJS, 123, 3

Marston, A. P., \& Appleton, P. N. 1995, ApJ, 109, 1002

Martin, C., \& Kennicutt, R. C. 2001, ApJ, 555, 301

Mihos, J. C., \& Hernquist, L. 1994, ApJ, 437, 611

Samland, M., Hensler, G., \& Theis, Ch. 1997, ApJ, 476, 544

Struck, C. 1997, ApJS, 113, 269

Struck, C., Appleton, P. N., Borne, K. D., \& Lucas, R. A. 1996, AJ, 112,1868

Struck-Marcell, C., \& Higdon, J. L. 1993, ApJ 411, 108

Vorobyov, E.I., Bizyaev D. 2001, A\&A, 377, 835

Vorobyov, E.I., Bizyaev D. 2003, A\&A, 400, 81 (VB)

Wada, K., \& Norman, C. A. 2001, ApJ, 547, 172 